\title{
De la sensation de l'odeur à la sensation du goût
}

Un miroitement des sensations et des images chez Odysseas Elytis et Nazim Hikmet

From Smell to Taste: A Mirroring of Sensations and Images in the Work of Nazim Hikmet and Odysseas Elytis

\section{Nadia Stylianou}

\section{OpenEdition}

Journals

Édition électronique

URL : https://journals.openedition.org/ceb/6584

DOI : $10.4000 /$ ceb.6584

ISSN : 2261-4184

Éditeur

INALCO

Édition imprimée

ISBN : 978-2-85831-230-6

ISSN : 0290-7402

\section{Référence électronique}

Nadia Stylianou, «De la sensation de l'odeur à la sensation du goût », Cahiers balkaniques [En ligne],

Hors-série | 2016, mis en ligne le 16 mars 2017, consulté le 06 juillet 2021. URL : http://

journals.openedition.org/ceb/6584; DOI : https://doi.org/10.4000/ceb.6584

Ce document a été généré automatiquement le 6 juillet 2021.

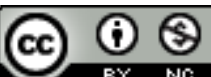

Cahiers balkaniques est mis à disposition selon les termes de la Licence Creative Commons Attribution - Pas d'Utilisation Commerciale 4.0 International. 


\title{
De la sensation de l'odeur à la sensation du goût
}

\author{
Un miroitement des sensations et des images chez Odysseas Elytis et \\ Nazim Hikmet \\ From Smell to Taste: A Mirroring of Sensations and Images in the Work of \\ Nazim Hikmet and Odysseas Elytis
}

Nadia Stylianou

1 Mon intervention sera axée sur deux poètes méditerranéens certes, mais d'origine différente : le poète grec, Odysseas Elytis et le poète turc, Nazim Hikmet dont j'essaierai de comparer l'esprit dans lequel ils ont créé leur vision du monde. Les deux poètes ont vécu pendant à peu près la même période: Elytis de 1911 à 1996 et Nazim Hikmet de 1902 à 1963. Tous deux ont combattu par leur œuvre pour la liberté et la dignité de l'homme.

2 Si nous focalisons notre regard sur la vie mouvementée des deux poètes, nous noterons qu'Elytis a vécu plusieurs grands moments de l'histoire de la Grèce moderne : la guerre déclarée à la Grèce par le fascisme italien aidé par la suite par les Allemands, l'occupation de la Grèce par les Allemands et les Italiens, la guerre civile entre nationalistes et communistes, le coup d'état des Colonels. De son côté, Nazim Hikmet paiera le prix de ses opinions politiques par quinze ans de prison, la perte de sa nationalité turque et un exil de douze ans en Union soviétique. Toutefois, il serait réducteur de lire leur poésie dans une optique strictement politique. L'art porte en soi l'universel, ainsi apparaîtra-t-il que la poésie d'Elytis comme celle de Nazim Hikmet est indéniablement humaniste et universelle.

Dans son essai À Livre Ouvert Elytis se réfère à Nazim Hikmet. Il y fait allusion aux poètes qui ont été influencés par le mouvement d'avant-garde artistique à Paris, mais qui ont pu, toutefois, préserver les traits distinctifs de leur culture et leur couleur locale.

Quant aux autres peuples, l'un plus, l'autre moins, ils étaient tous au centre de l'intérêt parisien sans pour autant perdre en rien leur couleur locale. Je trouverai un jour l'occasion d'expliquer combien, à mon avis, certains, sinon tous, ont profité avec des résultats beaucoup plus féconds de ces fermentations artistiques en 
France.

La voix fraîche et puissante de Vladimir Maiakovski s'élevant entre le futurisme et la fierté surréaliste contemporaine planait encore sur l'Europe, avec pour écho dans l'Europe du sud, le cri de protestation du poète turc Nazim Hikmet. ${ }^{1}$

Suivant le périple des goûts et des sensations on examinera quelle est la relation des deux poètes avec la langue, la nature, le sentiment métaphysique et l'amour.

\section{L'eau et le pain}

5 Un point commun d'inspiration chez les deux poètes dans leur démarche poétique est "l'eau » et «le pain », éléments qui renvoient à la notion du " peu ». La frugalité, la pauvreté, une réalité vécue chez Nazim, le « peu », choix d'Elytis dans sa philosophie et son attitude devant la vie, y sont vus comme élément de base de la culture grecque. Elytis, par son parcours et son acte, en fait un élément spiritualisé.

6 «Pain » est un des mots les plus fréquents chez Nazim Hikmet. Des graffitis sur le mur « pain et liberté » à ses vers fervents, il a une place privilégiée dans son œuvre. Le pain est le mot qui hante ses poèmes, associé au mot "vie», ainsi qu'à des substantifs accompagnés de «vivant» ou du verbe « vivre ». D'une part, «pain » mot concret et d'autre part, le mot "vie», renvoient à des constantes variées, il peut évoquer le quotidien mais aussi de multiples valeurs précieuses. Le pain en soi est une valeur suprême au nom de laquelle le moi poétique combat pour sa liberté.

7 Chez Nazim Hikmet, lors de son expérience de la prison, le pain se mue en mesure du temps, tel une aiguille d'horloge donnant au poète la possibilité de mesurer le temps.

\begin{tabular}{|c|c|}
\hline 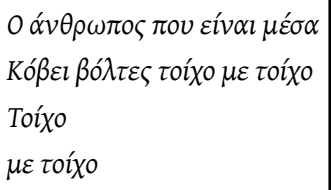 & $\begin{array}{l}\text { L'homme là-dedans marche } \\
\text { il va d'un mur à l'autre, } \\
\text { D'un mur } \\
\text { à l'autre }\end{array}$ \\
\hline 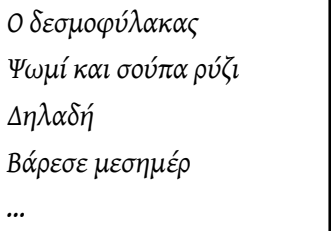 & $\begin{array}{l}\text { Le gardien } \\
\text { soupe au riz et pain } \\
\text { Donc : } \\
\text { midi a sonné } \\
\text {... }\end{array}$ \\
\hline 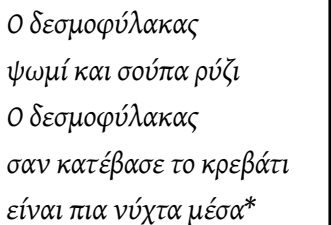 & $\begin{array}{l}\text { Le gardien } \\
\text { soupe au riz et pain } \\
\text { Quand le gardien } \\
\text { rabaisse le châlit } \\
\text { C'est la nuit là-dedans** }\end{array}$ \\
\hline
\end{tabular}

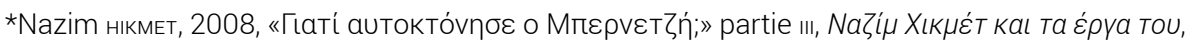
p. 303-304.

**Nazim нікмет, Livre premier et dernier, Pourquoi Benerdji s'est-il suicidé ? p. 95-96.

8 Les poèmes de combat de Nazim Hikmet courent, des rues d'Istanbul en Anatolie, d'Ankara à Bursa. Le pain est l'élément primordial de la survie : 


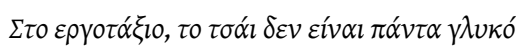
oú $\varepsilon \pi \alpha \dot{v} v \tau \alpha \zeta \varepsilon \sigma \tau o ́$

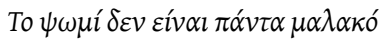

Au chantier, le thé n'est pas toujours sucré, ni toujours chaud

Le pain n'y est pas toujours tendre*

* Nazim HIKMEт, 2014, « Dans la sueur et dans le sang », I/ neige dans la nuit, p. 111.

9 Le cœur de Nazim Hikmet bat à l'écoute de tous les événements de chaque jour du monde, la guerre, Hiroshima, la mort.

\begin{tabular}{|c|c|}
\hline 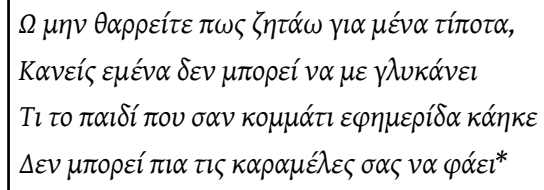 & $\begin{array}{l}\text { Pour ce qui est de moi } \\
\text { Je ne vous demande rien } \\
\text { il ne saurait manger, même des bonbons, } \\
\text { l'enfant qui comme du papier a brûlé** }\end{array}$ \\
\hline
\end{tabular}

*Nazim нікмет, 1966, Поıйната, р. 88

**Nazim Hікмет, « La petite fille», I/ neige dans la nuit, p. 115.

Chez Elytis le pain et l'eau constituent des éléments primordiaux menant à la

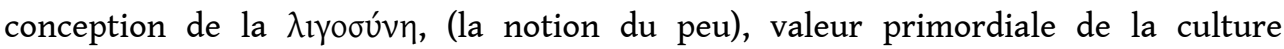
néohellénique. Dans l'essai À Livre Ouvert il écrit :

Souvent, il m'est arrivé d'éprouver un sentiment de malaise. Il me suffit alors, par exemple, de boire un peu d'eau fraîche et de manger du pain sec ou de penser très intensément à une île de l'Égée dans le soleil de l'été et le bruit des vagues pour retrouver mon équilibre avec un sentiment qui n'a rien à voir ni avec la métaphysique, ni avec l'autosuffisance esthétique, ni avec l'assurance d'une force matérielle. Mais je dirais comme une certitude qu'il existe une réserve de lumière suffisante au monde pour contrebalancer l'obscurité, et qu'en aidant à l'amener à la surface et seulement avec cela, nous parvenons à une harmonie avec l'existence qui accomplit son destin ${ }^{2}$.

Dans le Petit Navigateur il dit :

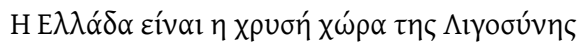

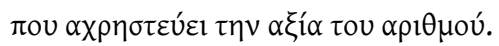

La Grèce est le pays par excellence de la notion du « peu »

qui annihile la valeur du nombre 3 .

Dans le même recueil il dit :

\begin{tabular}{|c|c|}
\hline 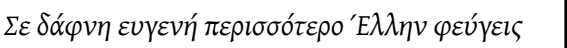 & Quelque noble laurier tu t'en vas plus Grec encore \\
\hline 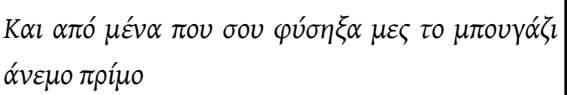 & $\begin{array}{l}\text { Que moi qui t'ai fait souffler un vent arrière dans le } \\
\text { détroit }\end{array}$ \\
\hline 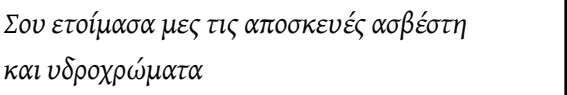 & $\begin{array}{l}\text { Et qui a préparé dans tes bagages de la chaux vive et } \\
\text { des détrempes }\end{array}$ \\
\hline 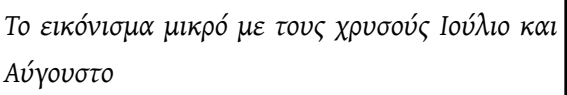 & $\begin{array}{l}\text { La petite image sainte avec, tout d'or vêtus, juillet et } \\
\text { août, }\end{array}$ \\
\hline 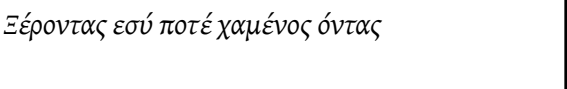 & $\begin{array}{l}\text { Sachant bien que lorsque à mon tour je } \\
\text { serai ce voyageur }\end{array}$ \\
\hline 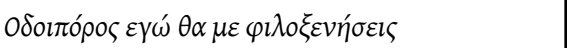 & Perdu tu me donneras l'hospitalité \\
\hline
\end{tabular}




\begin{tabular}{|c|c|}
\hline 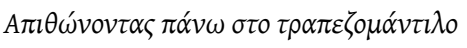 & Posant sur la nappe \\
\hline 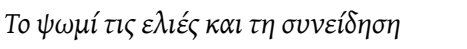 & Le pain les olives et la conscience \\
\hline 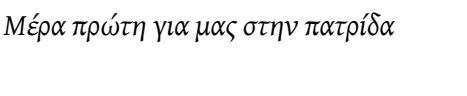 & $\begin{array}{l}\text { Premier jour pour nous dans votre } \\
\text { seconde patrie }\end{array}$ \\
\hline 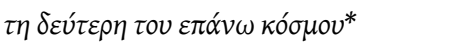 & La patrie du monde supérieur** \\
\hline
\end{tabular}

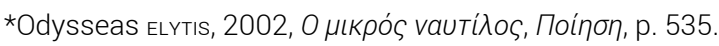

**Odysseas elytis, 1983, le Petit matelot, les Analogies de lumière, p. 52.

Le poète est en voyage constant. Dans ses bagages le moi poétique n'apporte que le nécessaire : la petite image sainte, objet sacralisé, ainsi que des détrempes et la chaux qui a une fonction purificatrice. Par la voie de la notion du peu, le moi poétique aboutit à l'autosuffisance, et par l'autosuffisance à une connaissance plus profonde de soimême. «La grandeur de la notion du peu se trouve dans le fait qu'elle rejette comme inconvenante et superflue la quantité et la richesse, en l'érigeant en un principe esthétique qui aiguise la capacité soustractive de façon que l'esprit ne conçoive que l'essentiel et le pertinent ${ }^{4}$ ». Le pain et les olives sont la métonymie du peu. Pour Elytis le « peu » est plus précieux que le tout. Si on remonte à Solomos, lui aussi met en relief deux traits pertinents de la tradition culturelle grecque, la pierre et l'herbe.

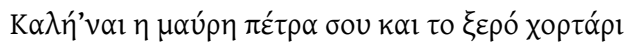

Bonne soit la pierre noire et l'herbe sèche

Ce vers revient chez Solomos comme un leitmotiv plus de trente fois dans des combinaisons expressives variées. Les deux éléments mentionnés sont apparemment insignifiants, mais ils constituent des éléments caractéristiques de la frugalité et de la simplicité. Cette frugalité et cette simplicité matérielle, ex principio négatives pour la vie de l'homme, puisque ces notions renvoient à la terre stérile, il les transmue en richesse spirituelle, en y voyant la source des valeurs culturelles. ${ }^{5}$

\section{Les poètes et les mots}

Les mots pour les deux poètes constituent une création en soi. Le langage poétique surgit de la matière, transgresse les conventions, brise la linéarité. Nazim Hikmet a recours à une métaphore concrète, palpable. Le Verbe (Kalâm), c'est-à-dire la parole, loin d'être uniquement le matériau de la création poétique, est son essence, il est la création même ${ }^{6}$. Julia Kristeva en marque fortement l'articulation: «Appelons métaphore, au sens général d'un transport de sens, de cette économie qui affecte le langage lorsque le sujet et l'objet de l'énonciation confondent leurs frontières ${ }^{7}$ ».

Dans un quatrain qui adopte la forme d'un rubaï Nazim Hikmet dit :

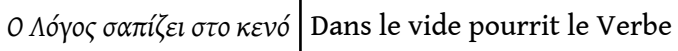




\begin{tabular}{|c|c|}
\hline$A v \delta \varepsilon \beta \gamma \alpha i v \varepsilon \varepsilon$ a $\alpha$ ó $\tau \eta \gamma \eta$ & S'il ne sort de la terre \\
\hline$A v \delta \varepsilon \beta v \theta i \zeta$ & S'il ne s'enfonce dans la terre \\
\hline 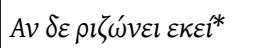 & S'il n'y ancre ses racines** \\
\hline
\end{tabular}

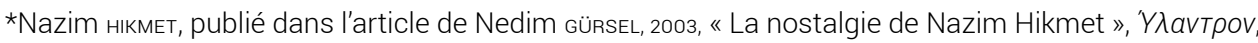
novembre, p. 102.

** Nazim HIKMET, publié dans l'article de Nedim GüRsEL, 2002, «Les mots de ma langue ressemblent à des pierres précieuses », Europe, numéro 878-879, juin-juillet, p. 96.

Nous rencontrons une image analogue chez Elytis :

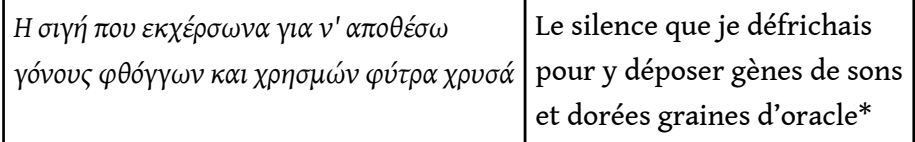

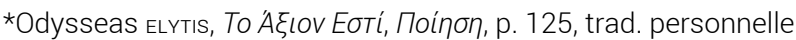

19 La création de la parole est, dans l'optique élytéenne, pareille à la culture de la terre. Le silence prend l'image d'une terre intangible où le poète va faire germer la parole poétique, «l'oracle doré », la révélation poétique.

À un deuxième niveau Nazim Hikmet nous révèle que la parole poétique, pour ne pas dépérir, pour être valable, doit s'enraciner dans la terre comme une plante qui a ses racines dans la terre. Elytis emploie une image semblable :

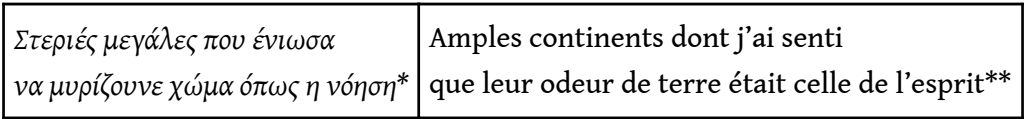

*Odysseas elytis, To Ákıov Eotí, ibid, p. 122.

**Odysseas elytis, 1987, Axion Esti, p. 56.

21 Pour Elytis, les sensations constituent le canal par la voie duquel la qualité de la nature influence l'esprit humain. La fonction mentale se forme en fonction de la qualité de la nature.

Dans un article publié en 1943 Nazim Hikmet écrit :

Les mots de ma langue ressemblent à des pierres précieuses. Des pierres précieuses, de couleur rouge, verte jaune, blanche, de toutes tailles, qui s'étalent devant mes yeux. Elles scintillent avec éclat... Mes yeux sont éblouis. Je les empoigne Elles glissent entre mes doigts, comme l'eau ensoleillée, brillante! Moi je suis un apprenti orfèvre. Je veux créer des sons inouïs en frappant les unes contre les autres...

Plusieurs années après avoir écrit ces lignes à Paris le poète, en regardant des tableaux de Matisse et d'Abidine, s'exclame :

Moi, les couleurs je les mange comme des fruits ${ }^{8}$

24 Nazim Hikmet est amoureux de sa langue, de ses couleurs et de ses saveurs, il aime créer des sons nouveaux qui provoqueront l'étonnement.

Elytis dit : 


\begin{tabular}{|c|c|}
\hline 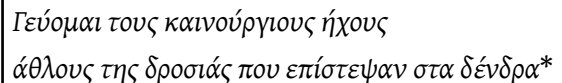 & $\begin{array}{l}\text { Je goûte les sons nouveaux et les combats de la rosée } \\
\text { qui firent confiance aux arbres** }\end{array}$ \\
\hline
\end{tabular}

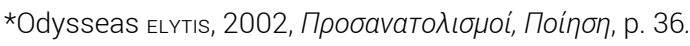

**Odysseas elytis, 1980, les Clepsydres de l'inconnu, Montpellier : fata morgana, p. 30, trad.

Jacques Lacarrière. notre regard sur la relation des deux poètes avec la nature nous constatons que la poétique de Nazim Hikmet fait écho à la philosophie orientale et évoque l'instinct dionysiaque de l'unité qui mène à l'absorption de l'individu dans des sphères élargies et dans les phénomènes de démission, d'absence, de fatalisme. Par contre, Elytis exprime l'équilibre entre la nature et la culture qui aboutit au prototype culturel néohellénique. Le modèle oriental dans ses manifestations extrêmes correspond au culte de la nature, à une conception mythique du Monde. À l'inverse, le modèle occidental dicte une démythification de la nature, une conception rationaliste du Monde qui favorise une attitude conquérante envers la nature. Elytis en exprimant le prototype culturel néohellénique révèle l'harmonisation de la nature et de la culture.

Dans "l'histoire du noyer et de Younous le boiteux », chez Nazim Hikmet, il y a comme une communication clandestine entre le moi poétique et le noyer, une réciprocité qui débouche sur un glissement des sensations et des sentiments.

\begin{tabular}{|c|c|}
\hline 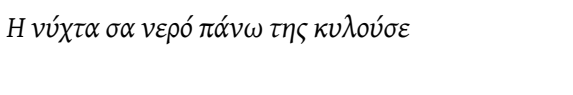 & $\begin{array}{l}\text { C'est une eau sombre qui ruisselle au-dessus de ta } \\
\text { tête }\end{array}$ \\
\hline 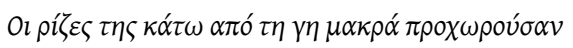 & Ses racines s'en allaient profond dans le sol \\
\hline 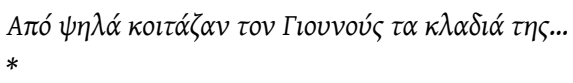 & Ses branches regardaient Younous d'en haut...** \\
\hline
\end{tabular}

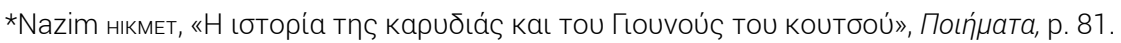

**Nazim HIKMEт, 2014, «L'histoire du noyer et de Younous le boiteux », // neige dans la nuit, p. 41.

Il y a une interpénétration constante de l'homme et de la nature. On a l'impression que les racines du noyer pénètrent dans l'âme de Younous, qui, toutefois, lui doit sa 
défaillance puisque «quand il est tombé d'une branche du noyer de là sa jambe qui boite » mais le noyer ne délivre pas son secret.

Par contre, dans la poésie élytéenne l'homme est un égal partenaire de la nature :

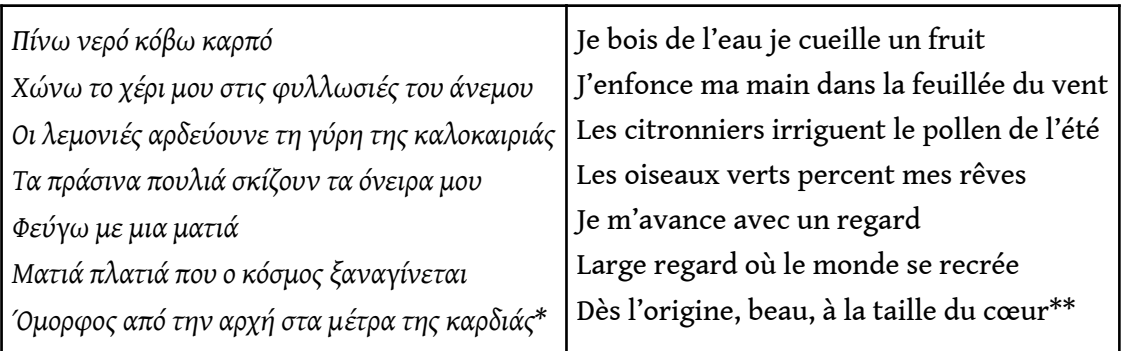

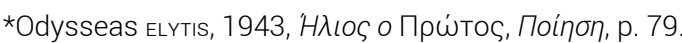

**Odysseas elytıs, Soleil Premier, Poèmes, 1945, p. 79.

Elytis se réfère aux arbres, aux plantes odorantes et il en extrait le sens caché et mystique par lequel ces constituants acquièrent des propriétés humaines. En même temps le poète s'approprie ces valeurs naturelles qui deviennent des valeurs morales. Il

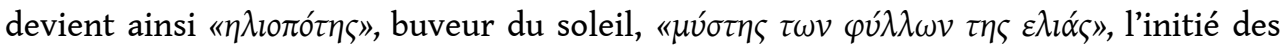
feuilles d'olivier. Dans le Petit Navigateur on lit :

\begin{tabular}{|c|c|}
\hline 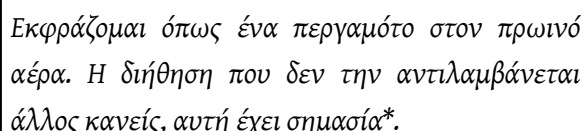 & $\begin{array}{l}\text { Je m'exprime comme une bergamote dans le vent } \\
\text { matinal. La distillation dont personne d'autre ne se } \\
\text { rend compte, c'est elle qui importe** }\end{array}$ \\
\hline
\end{tabular}

*Odysseas elytis, O

**Odysseas elytis, 2006, le Petit navigateur, L'Échoppe, p. 29

Ou dans Soleil Premier :

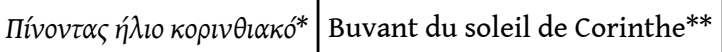

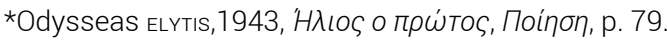

**Odysseas elytis, 1945, Poèmes, p. 79.

Par le verbe actif ici «buvant» le moi poétique absorbe les propriétés de la lumière, élément primordial de l'expression de la transparence. La lumière chez Elytis donne accès à la transparence. La transparence est ce don de voir à travers les choses, la capacité de voir à travers la surface extérieure des choses, d'unir le paysage physique au paysage spirituel dans une unité extrême. La transparence constitue une valeur morale que tout au long de sa vie le poète cherche à s'approprier : «La transparence qui existe au point de vue physique dans la nature se transfère à la poésie $»^{10}$. Nazim Hikmet a une imagerie semblable sur ce sujet : 


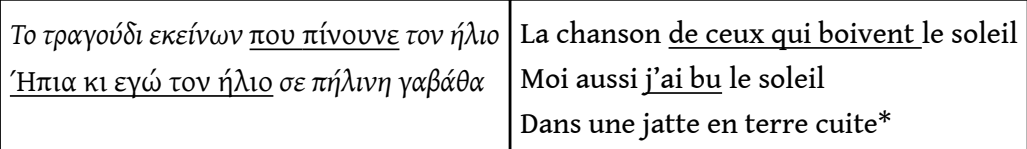

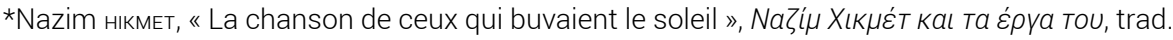
personnelle.

Le moi poétique chez les deux poètes devient le buveur du soleil, avec la différence que Nazim Hikmet parle, lui, de conquérir le soleil. Elytis élabore sa patrie transcendante, non pas dans le but d'identifier la réalité quotidienne à la réalité poétique, mais dans celui de leur faire acquérir entre elles des contacts étroits. Le grenadier en folie chez Elytis exprime la jouissance de la vie, le pouvoir de rêver, les orangers et les mandariniers mènent le poète à une communion avec le patrimoine spirituel, la pensée des anciens Ioniens. Elytis essaie de reconstituer cette Hellade imaginée, la seconde Hellade du monde supérieur, basée d'une part sur la géométrie de Platon et d'autre part sur un jardin peuplé d'arbres qui sentent Héraclite et Archiloque :

\begin{tabular}{|c|c|}
\hline 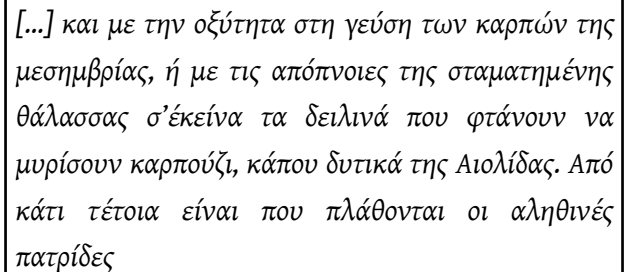 & $\begin{array}{l}\text { [...] avec l'acide dans le goût des fruits de midi ou } \\
\text { l'arrêt du souffle d'une mer immobile lors de ces } \\
\text { crépuscules qui sentent la pastèque quelque part à } \\
\text { l'ouest de l'Éolide. C'est à partir de choses } \\
\text { pareilles que se façonnent les vraies patries*. }\end{array}$ \\
\hline
\end{tabular}

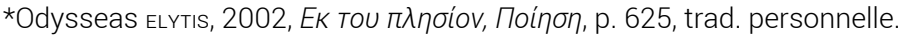

Le paysage offre ce qu'on peut nommer l'expérience proustienne. Ici ce n'est pas la saveur de la madeleine mais l'odeur de la pastèque qui amène le poète à l'autoconnaissance. L'Hellade d'Elytis est matérielle et spirituelle, chrétienne et païenne. Elle embaume de la senteur de pastèque en plein été mais en même temps, à un deuxième niveau, est peuplée d'arbres qui sentent Archiloque et Héraclite. Le poète veut faire paraître la seconde réalité qui se trouve à l'état latent sous la première. Or, Elytis intègre la Grèce au monde du rêve et la hausse au niveau du monde supérieur. "L'Hellade ne s'épuise pas seulement en un paysage, par contre, elle se transforme en un point de départ ou un prétexte pour que le poète arrive à créer son propre univers, l'Hellade du monde supérieur ${ }^{11}$ ». Ainsi le paysage devient-il une représentation idéale du monde véritable qui n'est pas toujours aussi clair, aussi vrai, aussi merveilleux, mais qui devrait et pourrait l'être.

37 La nature est hors du péché chrétien et la jouissance est pure. Il n'y a pas de faute originelle. Cela nous conduit à la problématique suivante: quelle est l'approche des deux poètes vis-à-vis du sentiment métaphysique? Dans leur interprétation métaphysique du monde les deux poètes ne distinguent pas le matériel du spirituel. Dans la mythologie poétique élytéenne le plaisir sensuel n'est pas un péché, même un ange peut en jouir sans culpabilité. Les anges jouissent sans remords de la saveur des fruits.

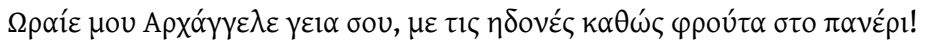

Salut bel Archange aux voluptés et aux fruits dans le panier !12 
s le monde habituel, l'Archange condamnerait conventionnellement les voluptés en tant qu'expression de la nature animale de l'homme, de ses instincts bestiaux. Elytis exempte même l'Archange des remords de la volupté en associant la jouissance des voluptés à l'assimilation des voluptés aux fruits. Il exalte la jouissance des valeurs naturelles sans aucune trace de connotation péjorative et par la nature même du sujet de l'action, de l'Archange, porteur des valeurs positives par excellence, qui, en y participant sanctifie les voluptés. L'Archange apparaît dans un univers uni, porteur des valeurs naturelles comme un bel adolescent qui tient et offre un panier de fruits, comme des voluptés. L'image donc annule la dualité puisqu'il humanise l'Archange, être métaphysique de la mythologie religieuse en un beau jeune homme ${ }^{13}$. Dans la mythologie élytéenne ce n'est pas seulement l'homme qui atteint le divin mais aussi le divin qui descend jusqu'à l'homme en lui devenant accessible, familier. Elytis recrée à partir du sentiment métaphysique un univers anthropocentrique en transposant l'élément métaphysique dans le monde physique. « De cette façon, le miracle se mue en une expérience quotidienne. Dans la mythologie de l'Égée le miracle se réalise dans la lumière et se caractérise par l'éblouissement de la révélation et non pas par la terreur métaphysique $»^{14}$.

Nazim Hikmet n'adopte pas la philosophie d'un " mysticisme cosmique» selon lequel il accorderait au monde une valeur absolue et rejoindrait Dieu à travers le monde sensible. Le Tawhid (l'Unité divine) est le dogme fondamental de l'Islam. «Dieu est unique, nul n'est semblable à lui, il n'est ni corps, ni individu, ni substance, ni accident. Il est au-delà du temps. Il ne peut habiter dans un lieu et dans un être, il n'est l'objet d'aucun des attributs ou des qualifications créaturelles. Il n'est ni conditionné ni déterminé, ni engendrant ni engendré. Il est au-delà de la perception du sens ${ }^{15}$. Or Nazim Hikmet est matérialiste. Il écarte l'hypothèse d'un créateur tout puissant en affirmant qu'il n'y a pas de cause première. Le monde n'est ni dessiné, ni créé. Nazim, en matérialiste, recourt aux sciences de la nature qui nous montrent que la terre existait déjà quand, ni l'homme, ni aucun être vivant en général ne l'habitait, ni ne pouvait l'habiter. Dans les deux premiers vers d'un rubaï ${ }^{16}$ il exprime la théorie darwinienne par une affirmation assez singulière ${ }^{17}$ :

Chou voiture microbe de la peste étoile

Nous sommes tous proches parents ${ }^{18}$

Ou encore, s'appuyant sur des images de la vie quotidienne il exprime son matérialisme d'une manière simple et pragmatique :

\begin{tabular}{|c|c|}
\hline 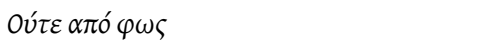 & Ni de lumière \\
\hline 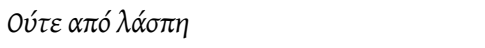 & ni de boue \\
\hline 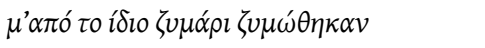 & mais de la même pâte sont pétris \\
\hline 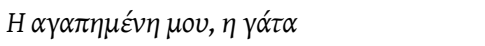 & ma bien aimée, sa chatte \\
\hline 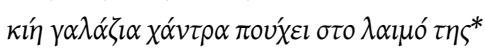 & et la perle bleue qu'elle porte au cou** \\
\hline
\end{tabular}

*Nazim нікмет, 1985, Поı́́ната, ibid., p. 129

**Nazim Hікмет, 2014, Il neige dans la nuit, p. 55.

Pour Nazim, le monde est un et il n'y a pas de culpabilité à jouir du monde matériel et sensuel, en particulier de l'amour. Décrivant l'acte érotique les deux poètes emploient 
des connotations gustatives en mettant en relief une dimension bipolaire de l'éros palpable et impalpable ; charnel et céleste, sexuel et sublime. Nazim Hikmet dit :

\begin{tabular}{|c|c|}
\hline$\Sigma^{\prime} \alpha \gamma \alpha \pi \omega^{\prime} o^{\prime} \pi \omega \varsigma \tau \rho \omega^{\prime} \omega \tau \sigma \quad \psi \omega \mu \mu^{\prime} \mu \varepsilon \tau o \alpha \lambda \alpha^{\prime} \tau l$ & Toi je t'aime comme je mange le pain avec du sel \\
\hline 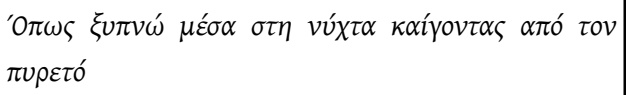 & $\begin{array}{l}\text { comme me réveillant dans la nuit brûlant de } \\
\text { fièvre }\end{array}$ \\
\hline 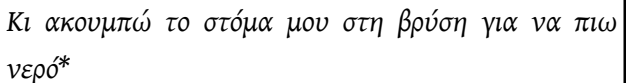 & $\begin{array}{l}\text { pour boire de l'eau j'appuie ma bouche au } \\
\text { robinet** }\end{array}$ \\
\hline
\end{tabular}

*Nazim Hікмет, 1980, Un étrange voyage, p. 184, trad. personnelle.

**Nazim нікмет, Un étrange voyage, ibid., p. 184. absence, situation en somme inouie : l'autre est absent comme référent, présent comme allocutaire. De cette distorsion singulière, naît une sorte de présent insoutenable [...]. L'Absence est la figure de la privation, tout à la fois, du je désire et du j'ai besoin. Le désir s'écrase sur le besoin : c'est là le fait obsédant du sentiment amoureux ${ }^{19}$. Elytis dit lui aussi :

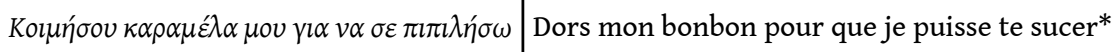

*Odysseas elytis, EK TOU $\pi \lambda \eta$ бíov, p. 595, trad. personnelle.

L'impératif « dors» sous-entend que le moi poétique tire de la jouissance par l'acte de son regard sur la femme aimée. Le moi poétique parfois se met à scruter le corps aimé (tel le narrateur devant le sommeil d'Albertine). Scruter veut dire fouiller, je fouille le corps de l'autre, comme si je voulais voir ce qu'il y a dedans ${ }^{20}$. La qualification de «bonbon » renvoie à la femme-enfant, notion chère aux surréalistes, car c'est celle qu'habite une jeunesse éternelle « parce que le temps sur elle n'a pas de prise $»^{21}$. 


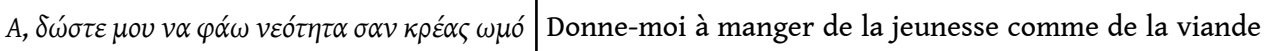

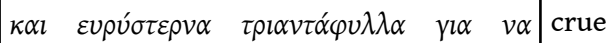

$\theta \omega \pi \varepsilon v ́ \sigma \omega^{*} \quad$ et des roses à la poitrine généreuse à caresser

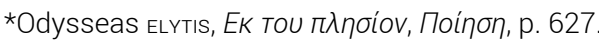

Il est significatif que ces vers véhiculent des expressions osées qui ont des connotations sexuelles. Partir de la sexualité va dans le sens d'un dévoilement et d'une lutte contre les interdits. Dans Malaise dans la civilisation Freud soutient que le refoulement accru de l'éros libère des pulsions destructrices. Ici le désir acquiert un caractère cannibale poussant l'amour jusqu'au délire en le chargeant d'un caractère dévorant et écrasant. Le caractère cannibale de l'éros nous le rencontrons également chez Nazim Hikmet :

\begin{tabular}{|c|c|}
\hline 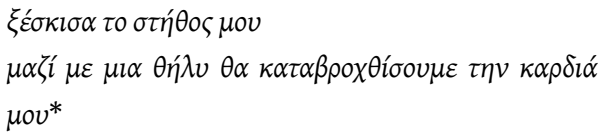 & $\begin{array}{l}\text { J'ai fendu ma poitrine } \\
\text { ensemble avec une femelle nous dévorons mon } \\
\text { cœur** }\end{array}$ \\
\hline
\end{tabular}

*Nazim Hikmet, 1980, « les Cannibales », Un étrange voyage, p. 177, trad. personnelle.

**Nazim Hikmet, « les Cannibales », Un étrange voyage, p. 177

Dans le jeu érotique les objets sexuels sont la cause « d'une alternance continuelle entre la répulsion et l'attraction, en conséquent de l'interdit et de la levée de l'interdit. Celui qui en consomme la chair n'ignore pas l'interdit dont cette consommation est l'objet $\aleph^{22}$. Le poète associe le verbe actif concret "manger» au nom abstrait « jeunesse ». Mais ici la jeunesse, loin de représenter pour le poète une période de la vie, est pour le poète une disposition d'esprit, dans et par laquelle on retrouve une authentique possession de soi- même. Or, la jeunesse est pour le poète un état d'esprit qu'il cherche à garder pour toujours :

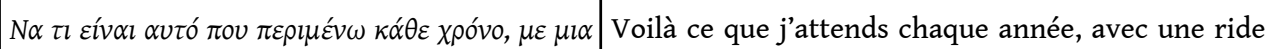

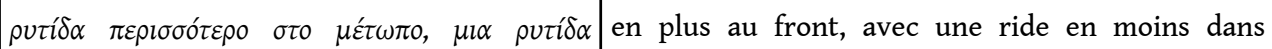

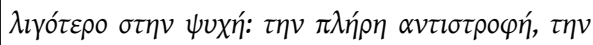

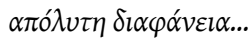
l'âme: Le retour à la jeunesse, la transparence absolue...*

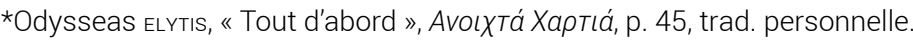

Par ce périple des goûts et des sensations chez les deux poètes nous avons étudié comment convergent et divergent l'esprit dionysiaque et apollinien, l'outrance de l'Orient et la notion du peu, le rationalisme occidental et le mysticisme de l'Orient. À travers ce bref parcours nous avons repéré certains traits distinctifs. Odysseas Elytis, exprimant le prototype culturel néohellénique et Nazim Hikmet, exprimant le prototype culturel de l'Anatolie. On aura remarqué que leurs sujets d'inspiration sont communs venant des pays méditerranéens. Odysseas Elytis, dans une interview, déclare sa conviction qu'il existe une sorte d'analogie entre la vision surréaliste et la vision orientale, spécialement islamique ${ }^{23}$. Elytis croit également qu'il existe chez les Grecs un aspect oriental qu'il ne faut pas négliger. D'après Elytis, l'élément oriental a toujours occupé une place importante dans la mentalité grecque ${ }^{24}$. Les deux poètes ont vécu à la même période dans des pays en proie à des conflits d'idéologie politique. Nazim Hikmet 
a plongé dans la lutte, il a souffert dans son intégrité morale pour ses idées sociales libérales. Elytis s'est mis en exil volontaire à Paris lors de la dictature des Colonels, jugeant de loin les aléas de la politique de la Grèce. Cependant, on retrouve les mêmes sources d'inspiration, leur philosophie est basée sur le choix des choses essentielles du «peu». Elytis en tire une philosophie et Hikmet une morale de lutte en faveur des pauvres et des persécutés. Poètes tous deux, ils révèrent le mot qui, pour les deux, est une création en soi. Hikmet révère le mot comme une création qui a ses racines dans la terre. Tous deux ont une relation très forte avec la nature. Nazim Hikmet voit l'homme et la nature mêlés l'un à l'autre. L'homme est absorbé. Chez Elytis il y a une équivalence des valeurs naturelles et des valeurs morales car Elytis bâtit son univers poétique sur la théorie des analogies. Il y a une équivalence entre la nature et l'esprit, le monde naturel et le monde spirituel. L'homme et sa culture se forment essentiellement à partir de l'expérience du monde comme le conçoivent les sensations. Elytis humanise la nature, en extrait le sens caché, la rend transparente et la hausse au niveau du monde supérieur.

50 La nature est hors du péché chrétien et la jouissance est pure. Le divin chez Elytis devient tangible et quotidien et l'humain atteint le divin. Nazim Hikmet est matérialiste et pragmatique. Il met en cause le principe du monde, rien n'est créé ou dessiné. Quant à l'amour, tous deux en font une force à la fois charnelle et spirituelle, une force de la nature sans péché, sans remords, mais y voient aussi un certain cannibalisme de l'homme pour retrouver la force, le goût pour la jeunesse éternelle. Les deux poètes se rejoignent dans leur rapport à la mer, élément fortement présent du monde méditerranéen et à jamais éternel, dont le mouvement est associé aux hauts et aux bas de la vie ; Nazim Hikmet dans « la mer Caspienne » dit :

\begin{tabular}{|c|c|}
\hline 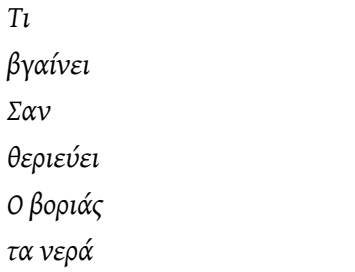 & $\begin{array}{l}\text { Qu'est-ce } \\
\text { que ça fait } \\
\text { que le } \\
\text { vent du } \\
\text { Nord } \\
\text { déchaîne les vagues }\end{array}$ \\
\hline 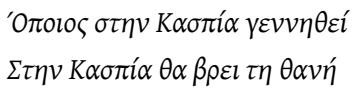 & $\begin{array}{l}\text { Qui voit le jour dans la mer Caspienne } \\
\text { dans la Caspienne mourra }\end{array}$ \\
\hline 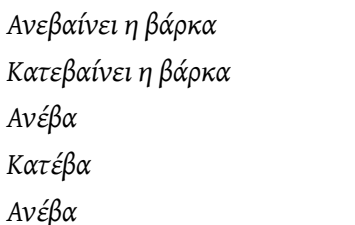 & $\begin{array}{l}\text { La barque monte } \\
\text { La barque descend } \\
\text { Monte } \\
\text { Descends } \\
\text { Monte* }\end{array}$ \\
\hline
\end{tabular}

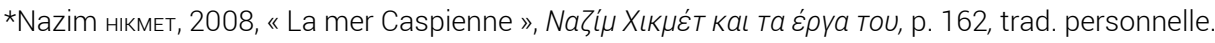

51 La vaste mer, l'immense mer salée, avec ses vagues et ses barques. La mer se métamorphose en combattant, le Pont-Euxin en la vie même, les vagues en hauts et en bas dont la vie est pleine ${ }^{25}$. Ces vers n'enclosent pas seulement la grandeur de la beauté esquissée par l'image du mouvement et de l'expression mais aussi la grandeur du contenu combatif et idéologique. Tu monteras en combattant sur les vagues de la vie, 
de la lutte, tu descendras, tu tomberas... car il n'est pas possible de dissocier le poète du lutteur.

Pour Nazim Hikmet la vie même est un mouvement perpétuel de haut en bas et de bas en haut, pareil à celui des vagues de la mer qui vont et viennent en résonnant de la philosophie d'Héraclite : tout change, tout s'écoule. La fluidité de la mer est illustrée par l'image de la vie même avec ses ondoiements. Aucune force humaine ne peut arrêter le mouvement des vagues car la mer « est toujours changeante et nous ne pouvons revivre deux fois le même moment car nous changeons aussi, au mobile correspond le mobile ${ }^{26} »$.

\begin{tabular}{|c|c|}
\hline 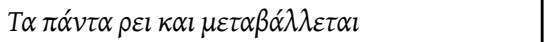 & « Tout change et s'écoule, \\
\hline 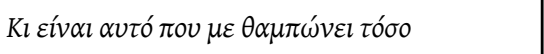 & Voilà ce qui me frappe et m'étonne » \\
\hline$\ldots$ & $\ldots$ \\
\hline 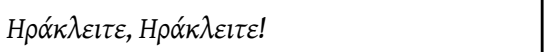 & Héraclite, Héraclite! \\
\hline 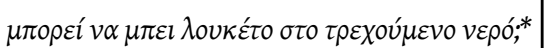 & Peut-on mettre sous clé l'eau qui coule ?** \\
\hline
\end{tabular}

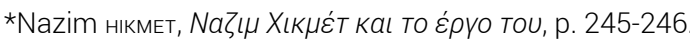

**Nazim нікмет, Pourquoi Benerdji s'est-il suicidé ?, p. 30.

53 À travers la fluidité des choses et du temps se révèle la notion de la durée. Dans le Petit

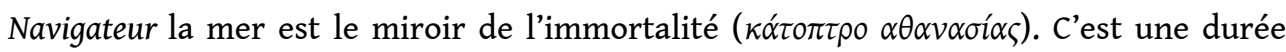
dont la lumière est si éblouissante qu'elle nous empêche de l'appréhender ${ }^{27}$. L'infinité de la mer débouche sur l'infinité du temps ${ }^{28}$ à travers lequel le poète navigue à hue et à dia pour découvrir qui il est.

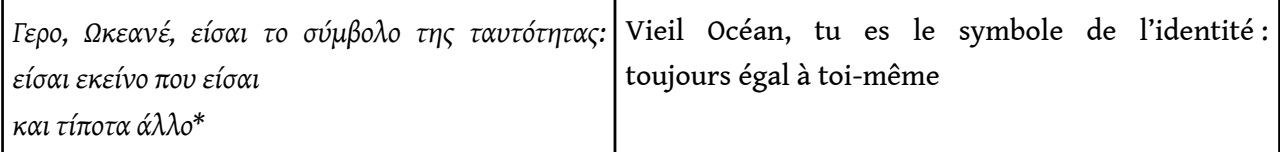

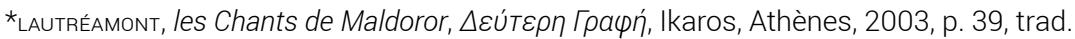

Odysseas Elytis.

54 Il ne faut pas oublier que les voix poétiques d'Odysseas Elytis et de Nazim Hikmet se nourrissent d'une tradition nationale et des traits distinctifs de leur culture, tels des torrents bouillonnants « avant d'aller se jeter dans d'autres fleuves dans cet immense océan verbal où s'enracine la culture humaine ${ }^{29}$.

Les poètes voyagent

sur les mers des univers

se regardant les uns les autres ${ }^{30}$

dira Nazim Hikmet dans son long et étrange voyage. 


\section{BIBLIOGRAPHIE}

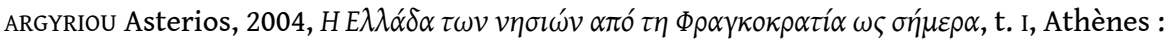

Hellinica Grammata.

AXELos Kostas, 2008, Héraclite et la philosophie, Paris : Les Éditions de Minuit.

BARTHES Roland, 1977, Fragments d'un discours amoureux, Paris : Éditions du Seuil.

BATAILLE Georges, 1992, l'Érotisme, Paris : Les Éditions de Minuit.

CORBIN Henry, 2014, Histoire de la philosophie islamique, Paris : Gallimard, Folio.

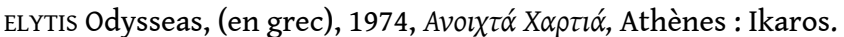

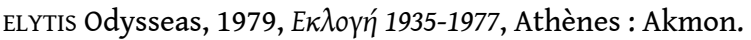

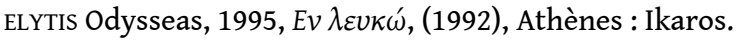

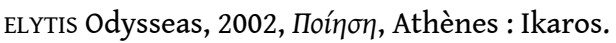

ELYTIS Odysseas, (en français), 1945, Poèmes, Athènes : Hestia, trad. Robert Levesque.

ELYTIS Odysseas, 1983, les Analogies de lumière, Marseille : Sud, trad. Jacques Phytilis.

ELYTIS Odysseas, 1987, Axion Esti, Paris : Gallimard, trad. Xavier Bordes et Robert Lonqueville.

ELYTIS Odysseas, 2006, le Petit navigateur, L'Échoppe, trad. Malamati Soufarapis.

FREUD Sigmund, 1971, Malaise dans la civilisation, Paris : P.U.F., trad. C. J. Odier.

GÜRSEL Nedim, 1987, Nazim Hikmet et la littérature populaire turque, Paris : Harmattan.

HIKMET Nazim, (en français), 1980, Un étrange voyage, Paris : François Maspéro, trad.

Munevver Andor et Guizine Dino.

HIKMET Nazim, 2005, Pourquoi Benerdji s'est-il suicidé ? Saint Gilles (B) : Éditions Aden.

HIKMET Nazim, 2014, Il neige dans la nuit, Paris : Gallimard.

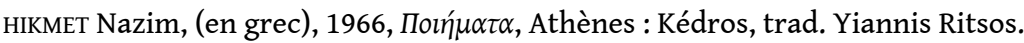

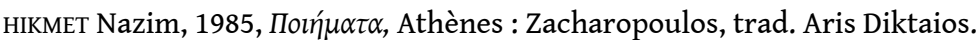

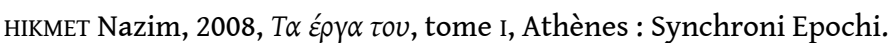

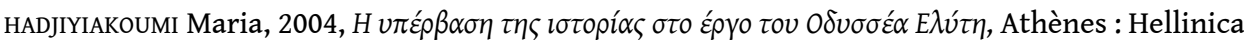

Grammata.

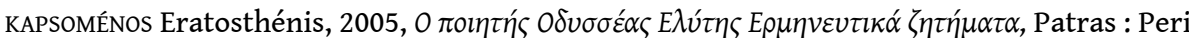

Technon, Patras.

\section{Revues en grec}

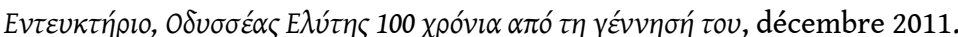

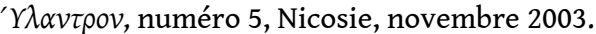

Revues en français

Digraphe, numéro 30, juin, 1983. 
Europe, revue consacrée à Nazim Hikmet, numéro 878-879, juin-juillet 2002.

Europe, revue consacrée à Nazim Hikmet, numéro 547-548, novembre-décembre 1974.

\section{NOTES}

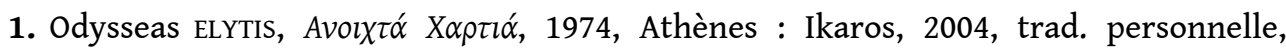
p. 124-125.

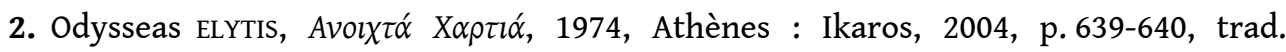
personnelle.

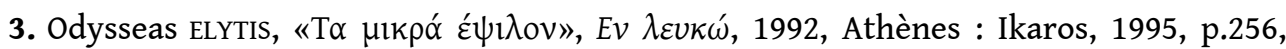
trad. personnelle.

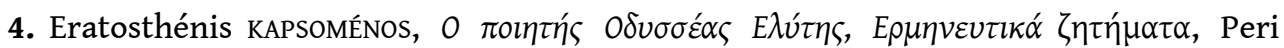
technon, Patras, 2005, p. 103, trad. personnelle.

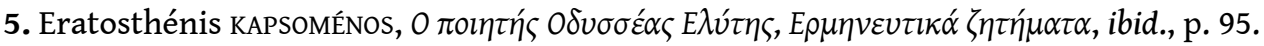

6. Nedim GÜRSEL, «Les mots de ma langue ressemblent à des pierres précieuses ", Europe, numéro 878-879, juin-juillet 2002, p. 96.

7. Julia KRISTEVA, Histoires d'amour, Denoël, 1983, p. 252.

8. Nazim HIKMET, « Un étrange voyage », Il neige dans la nuit, ibid., p. 209.

9. Paule Plouvier, Poétique et analytique de l'amour dans l'œuvre d'AndréBreton, thèse, Université de Vincennes, Paris VIII, 1960, p. 338.

10. Odysseas ELYTIS, les Analogies de lumière, interview avec Ivar Ivask, Marseille : éd. Sud, 1983, p. 100, trad. Jacques Phytilis.

11. Paola Maria MINUCCI, «La Grèce au-delà de la Grèce dans la poésie

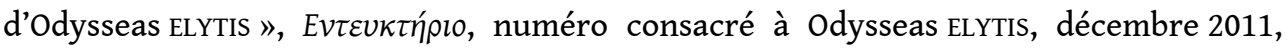
p. 17.

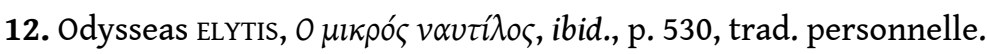

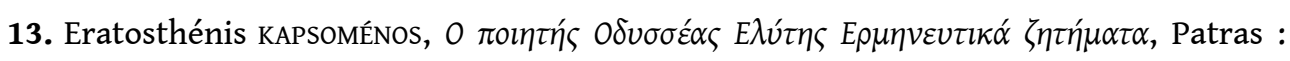
Peri Patron, 2005, p. 112-113, trad. personnelle.

14. Eratosthénis KAPSOMÉNOS, «La mythologie de l’Égée dans la littérature

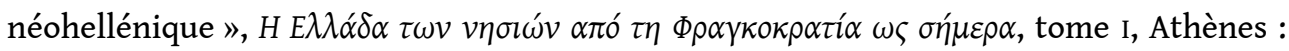
Hellinica Grammata, 2004, p. 409.

15. Henry CORBIN, Histoire de la philosophie islamique, Paris : folio, 2014, p. 161.

16. Note: Le rubaï est une forme arabo-persane que seuls les grands poètes ont su maîtriser. Employé de préférence pour exprimer une pensée philosophique, il est composé de quatre vers dont les deux premiers et le dernier riment entre eux. C'est une des rares formes dans la poésie orientale où le thème se développe au lieu de s'éparpiller en variation.

17. Nedim GÜRSEL, Nazim Hikmet et la littérature populaire turque, Paris : L'Harmattan, 1987, ibid. p. 87.

18. Nazim HIKMET, Il neige dans la nuit, ibid., p. 55.

19. Roland BARTHES, Fragments d'un discours amoureux, Paris : Seuil, 1977, p. 21-22. 
20. Roland BARTHES, Fragments d'un discours amoureux, ibid., p. 85.

21. André BRETON, Arcane 17, éd. J. J. Pauvert, 1971, p. 71.

22. Georges BATAILlE, l'Érotisme, Paris : Les Éditions de Minuit, 1992, p. 80.

23. Odysseas ELYTIS, Digraphe, juin 1983, p. 116.

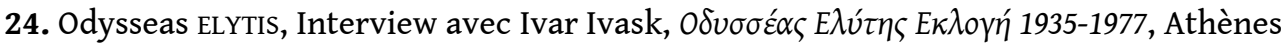
: Akmon, 1979, p. 202, trad. personnelle.

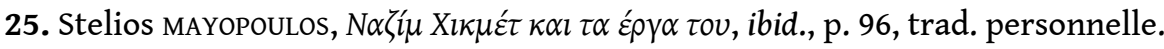

26. Kostas AXELOS, Héraclite et la philosophie, Les Éditions de Minuit, 1980, p. 30.

27. Odysseas ELYTIS, le Petit Navigateur, ibid., p. 33.

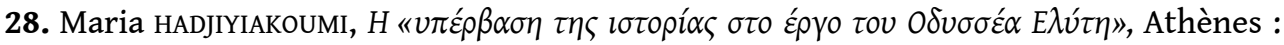
Hellinica Grammata, 2004, p. 114, trad. personnelle.

29. Nedim GÜRSEL, «Poésie populaire et poésie du divan», Nazim Hikmet Europe, numéro 547-548, novembre-décembre, 1974, p. 35.

30. Nazim HIKMET, « Le voyage », Un étrange voyage, ibid., p. 173.

\section{RÉSUMÉS}

Cette communication est axée sur deux poètes méditerranéens, mais d'origine différente, Odysseas Elytis (poète grec) et Nazim Hikmet (poète turc), dans le but de comparer leur vision du monde. Notre cheminement de réflexion se focalisera sur la relation des deux poètes avec la langue, la nature, le sentiment métaphysique et l'éros. À travers ce périple des goûts et des sensations, on étudiera comment convergent et divergent l'esprit dionysiaque et apollinien, l'outrance de l'Orient et la notion du peu, le rationalisme occidental et le mysticisme d'Orient. On essaiera également de repérer les traits distinctifs dans leur œuvre, celle d'Elytis exprimant le prototype culturel néo-hellénique et celle de Nazim Hikmet exprimant celui de l'Anatolie. Toutefois, on décèle aussi une certaine tonalité orientale dans la vision et la mentalité grecques.

This paper focuses on two Mediterranean poets of different backgrounds, namely the Greek poet Odysseas Elytis and the Turkish poet Nazim Hikmet, aims at comparing their divergent worldviews. Our exploration will focus on the poets' relationship with language, nature, the metaphysical feeling and Eros. Through this circumnavigation of flavours and sensations we will study how the Dionysian and the Apollonian spirit, the opulence of the East and the concept of the minimal, Western rationalism and Eastern mysticism converge and diverge. At the same time, we will attempt to identify those distinctive features that express the contemporary Greek cultural model in the work of Odysseas Elytis and the oriental spirit in the work of Nazim Hikmet. We will also trace a certain resonance from Eastern philosophy within the Greek mindset and vision.

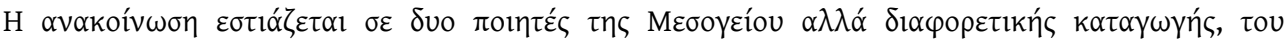

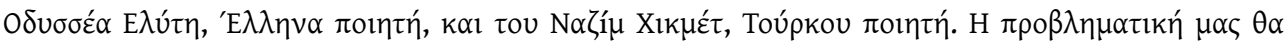

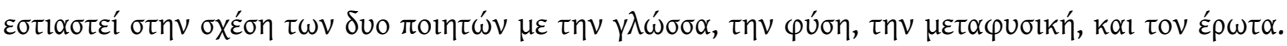

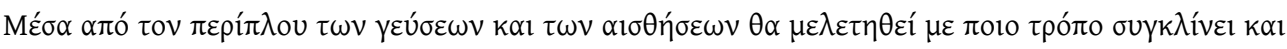




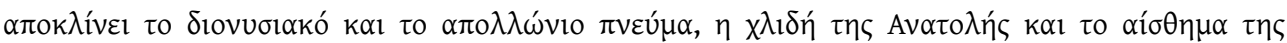

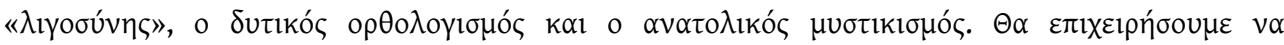

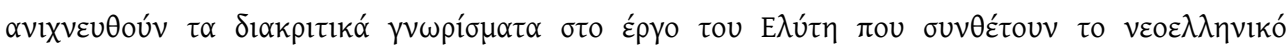

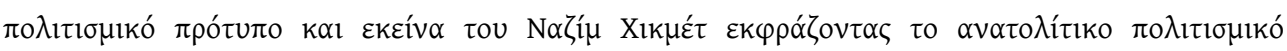

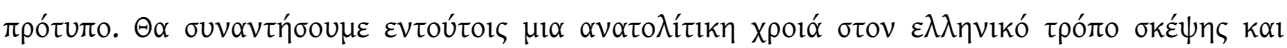

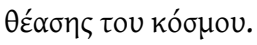

\section{INDEX}

motsclestr Elitis Odisseus (1911-1996), Hikmet Nazim (1901-1963), Kültür, Doğa, Kimlik, Eros, Akdeniz, Yunanistan, Türkiye, Yirminci yüzyıl, Edebiyat, şiir Mots-clés : culture, culture, nature, nature, frugalité, frugalité, mysticisme, mysticisme, rationalisme, rationalisme, identité, identité, éros, éros, Elytis Odysseas (1911-1996), Elytis Odysseas (1911-1996), Hikmet Nazim (1902-1963), Hikmet Nazim (1902-1963)

Thèmes : Littérature, Poésie

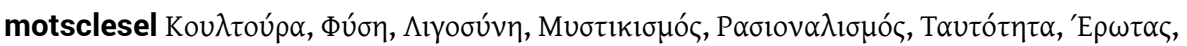

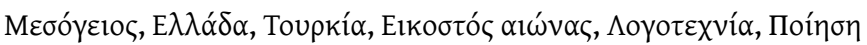
motsclesmk ЕЛИТИС ОДИСЕЈ (1911-1996), ХИКМЕТ НАЗИМ(1901-1963), КУЛТУРАТА, ПРИРОДАТА, МИСТИЦИЗАМ, РАЦИОНАЛИЗАМ, ИДЕНТИТЕТ, ЕРОС, МЕДИТЕРАНОТ, ГРЦИЈА, ТУРЦИЈА, ДВАЕСЕТТИОТ ВЕК, ЛИТЕРАТУРА, ПОЕЗИЈА

Index géographique : Méditerranée, Grèce, Turquie

Keywords : Elytis Odysseas (1911-1996), Hikmet Nazim (1902-1963), Culture, Nature, Frugality, Mysticism, Rationalism, Identity, Eros, Mediterranean, Greece, Turkey, Twentieth century,

Literature, Poetry

Index chronologique : vingtième siècle

\section{AUTEUR}

\section{NADIA STYLIANOU}

Université de Chypre 